\title{
Plataformas virtuales como herramientas de enseñanza
}

\author{
Virtual platforms as teaching tolos
}

Plataformas virtuais como ferramentas de ensino.

\section{ARTÍCULO GENERAL}

Miryam Maria del Rosario Ramos Vite

https://orcid.org/0000-0001-9437-9696

mramosv8@ucvvirtual.edu.pe

Universidad César Vallejo

\author{
Luz Carola Macahuachi Nuñez de Castillo \\ https://orcid.org/0000-0002-8879-7637 \\ Ideca28@ucvvirtual.edu.pe \\ Universidad César Vallejo
}

Recibido 01 de Diciembre 2021 | Arbitrado y aceptado 01 de Diciembre 2021 | Publicado en 24 Enero 2022

\section{RESUMEN}

Las plataformas virtuales se han convertido hoy en día en herramientas indispensables en el proceso de enseñanza aprendizaje. Asimismo, la sociedad de hoy exige que la educación pueda ser adaptada a su realidad con los elementos con que cuenta. Por tal razón se realizará una revisión sobre las plataformas virtuales como método de enseñanza tomando en cuenta la realidad de los docentes y los resultados de su uso en diferentes modalidades. Para ellos consultaremos con diferentes artículos nacionales e internacionales que nos muestran sus realidades, ventajas y desventajas de la utilización de estas herramientas, con el fin de evaluar y mejorar los aprendizajes asíncronos en los que nos hemos visto forzados a llevar a consecuencia de la pandemia del COVID 19.

Palabras clave: Plataforma virtual, enseñanza aprendizaje, tecnologías

\section{ABSTRACT}

Virtual platforms have today become indispensable tools in the teachinglearning process. Likewise, today's society demands that education can be adapted to its reality with the elements it has. For this reason, a review will be carried out on virtual platforms as a teaching method, taking into account the reality of teachers and the results of their use in different modalities. For them, we will consult with different national and international articles that show us their realities, advantages and disadvantages of the use of these tools, in order to evaluate and improve the asynchronous learning in which we have been forced to carry out as a result of the coronavirus pandemic. COVID 19.

Keywords: Virtual platform, teaching learning, technologies

\section{RESUMO}

As plataformas virtuais tornaram-se hoje ferramentas indispensáveis no processo de ensino-aprendizagem. Da mesma forma, a sociedade atual exige que a educação se adapte à sua realidade com os elementos que possui. Por esse motivo, será realizada uma revisão em plataformas virtuais como método de ensino, levando em consideração a realidade dos professores e os resultados de sua utilização em diferentes modalidades. Para eles, vamos consultar diferentes artigos nacionais e internacionais que nos mostram suas realidades, vantagens e desvantagens do uso dessas ferramentas, a fim de avaliar e melhorar a aprendizagem assíncrona em que fomos obrigados a realizar como resultado de a pandemia de coronavírus. COVID 19.

Palavras chave: Plataforma virtual, ensino aprendizagem, tecnologias 


\section{Introducción}

El año 2020 va a pasar a la historia por la pandemia mundial debida al virus SARS-CoV-2, causante de la enfermedad COVID-19. Es así, como la incorporación de los medios digitales ha implicado una transformación a nivel mundial en diferentes ámbitos: educativo, económico, social, político y cultural (Organización para la Cooperación y el Desarrollo Económicos, OCDE, 2015). La propagación de las tecnologías de la información y de la comunicación (TIC) son notorias en prácticamente todos los aspectos de la actividad de las personas, además de multiplicar las posibilidades y los contextos de aprendizaje más allá del escenario formal (Cabero, 2017; Coll y Monereo, 2008).

El Ministerio de Educación del Perú, estableció normas ante esta situación que han venido siendo modificadas en constantes oportunidades, ya que, hasta la fecha de este escrito, seguimos el proceso educativo a distancia. En un inicio la Resolución Viceministerial N 097-2020-MINEDU, estableció las "Disposiciones para el trabajo remoto de los profesores que asegure el desarrollo del servicio educativo no presencial de las instituciones y programas educativos públicos, frente al brote del COVID-19”. A esta resolución se acogen todas las instituciones educativas de Educación Básica Regular, CEBA, EBE y PRONOEI. Esta resolución aborda varios puntos, desde los procesos de admisión, matrícula, pasando por la planificación curricular, reprogramación y recuperación de clases, entre otros puntos necesarios que permitan contar con las condiciones para otorgar el proceso formativo. Así como dentro de la implementación del servicio educativo, nos menciona que podemos utilizar varios métodos no presenciales mediante correo electrónico, llamada telefónica, portal web institucional u otras que establezca la institución, así como el apoyo de los docentes de computación e informática sobre el acceso y el uso de la plataforma, aula virtual u otro medio determinado para el desarrollo de la modalidad no presencial. Para ello sugiere la misma norma utilizar material audiovisual instructivo y canales de soporte técnico.

Por tanto, las TIC configuran nuevos entornos y escenarios para la formación inicial docente en la orientación y tutorización, eliminando barreras espacio-temporales y facilitando el trabajo colaborativo y la flexibilidad en el aprendizaje (Domingo y Marqués, 2011). Algunas investigaciones buscan comprender las potencialidades del uso de las TIC en la entrega de retroalimentación formativa (Remesal, Colomina, 
Mauri, y Rochera, 2017) para ayudar a los estudiantes a realizar trabajos de forma colaborativa (Mauri, Ginesta \& Rochera, 2016) y en la evaluación de aprendizajes de estudiantes, favoreciendo la autorregulación en el proceso educativo.

Los ambientes virtuales en el contexto de la educación son espacios de interacción entre docentes y estudiantes, espacios donde se facilita la comunicación, la didáctica y la pedagógica, para intercambiar recursos didácticos textuales, multimediales e interactivos. Este espacio está disponible en todo momento para estudiantes y docentes, creando así un espacio de intercambio de información y actividades de aprendizaje, estos ambientes llamados virtuales por estar mediados por la tecnología son un apoyo tanto para la educación presencial, distancia (ahora virtual) y semipresencial. (Muñoz, García y Rodriguez, 2011).

Asimismo, García (2017) en su tesis doctoral Competencias digitales en la docencia universitaria del siglo XXI en la Universidad Complutense de Madrid, Facultad de Educación, nos menciona que una de las competencias que deben contar los docentes universitarios es la competencia digital, esta implica el uso de las tecnologías de la información y el conocimiento (TIC) dentro y fuera del aula, ya que permiten la conexión del alumno a las plataformas virtuales, así como realizar el seguimiento de las actividades programadas por el profesor en todo momento. Y aunque recién se están realizando los estudios al respecto, sí se observa una relación directa entre el uso de las TIC, incluyendo a la plataformas virtuales y resultados positivos en cuanto a la mejora de la comprensión de los contenidos. Lo que sí es importante, recalca el autor, es que nadie pone en tela de juicio es la vital importancia de que los estudiantes deben adquirir esta competencia digital ya que será sumamente necesario y será una demanda para su incorporación al mundo laboral.

Una de las implementaciones más robustas y exhaustivas de las TIC en la educación se realiza por medio de los sistemas de gestión del aprendizaje (SGA). Estos permiten crear aulas virtuales, donde el trabajo del profesor es facilitar el aprendizaje a partir de temas, recursos y actividades previamente seleccionados para impartir sus asignaturas. Los SGA permiten presentar un curso ajustado a las necesidades de los alumnos para quienes el uso de teléfonos celulares, tabletas y laptops es cotidiano. Por lo tanto, es necesario probar y evaluar las plataformas disponibles para aprovechar estos dispositivos en la educación, ya que ayudan al proceso de enseñanza-aprendizaje (May, Patrón y Sahuí, 2017). 
En el artículo Memorias del Programa de Redes-I3CE de calidad, innovación e investigación en docencia universitaria, Convocatoria 2016-17, dado en España, se menciona que el mundo es cada vez el mundo está más digital y que las diferentes plataformas facilitan el aprendizaje en línea para los estudiantes, así como que el objetivo de estas herramientas es ayudar a los profesores a ahorrar tiempo, organizar todas sus clases y mejorar la comunicación con los estudiantes. Cooperberg (2002).

Dicha adaptación requiere que los docentes cuenten con las habilidades para actuar como guía y facilitador en el proceso de enseñanza aprendizaje, así como también para integrar las tecnologías de información a sus métodos de enseñanza. Según Puentes, Roig, Sanhueza y Friz (2013), la incorporación de las TIC está relacionada con dos ideas centrales: 1) el papel de las tecnologías en el proceso de socialización; y, 2) su vinculación con el proceso de aprendizaje y que, en consecuencia, lleva a situar el fenómeno educativo en un contexto social donde surge la necesidad de desarrollar competencias que permitan la selección y utilización adecuada de la información, lo que implica definir los roles del educador y educando.

La idea de vincular las TIC con el proceso de aprendizaje y la necesidad de que el docente desarrolle, adquiera y recicle nuevas competencias, están relacionadas, por un lado, al ámbito de la formación del profesorado, y por otro, al empleo educativo de las TIC, donde, según Cabero, Marín y Castaño (2015) se han encontrado dos problemas fundamentales. En primer lugar, mencionan la excesiva tecnificación en torno a los cursos que se diseñan y llevan a cabo; y, en segundo, señalan la escasez o falta de modelos conceptuales para dirigir la capacitación del docente (Monzoy, Acosta y Sanchez, 2011), la cual se supone, deberá proveer al profesor de los conocimientos, habilidades, recursos y destrezas necesarias para integrar de manera eficaz éstas en la práctica educativa y/o profesional.

En virtud de lo mencionado anteriormente queremos, en este artículo, opinar sobre artículos referentes a las Herramientas digitales en la educación. Por ello estaremos mencionando cinco artículos entre nacionales y extranjeros, tomando en cuenta los siguientes puntos esenciales al dialogar sobre el tema:

- Las plataformas virtuales en la educación.

- Los docentes frente a las herramientas digitales.

- Efectividad de las plataformas virtuales en el proceso de enseñanza aprendizaje. 


\section{Metodología}

El primero a tomar en cuenta es el artículo del autor Claro (2017) de Colombia, Valoración del uso de la plataforma virtual Moodle como recurso pedagógico en la enseñanza universitaria de la informática, nos abre el panorama del uso de una plataforma, en este caso Moodle. Se eligió este artículo tomando en cuenta las dificultades que se presentaron en cuanto a la presentación de la plataforma, el seguimiento que se debe hacer a los estudiantes para poder utilizarla y por último el tema de los docentes de ser reemplazados en su labor como profesores de cátedra, con la eventual pérdida de su empleo, temores que se vienen generando en algunos docentes mirando el futuro virtual.

Leiva y López (2019) de Chile, este artículo fue seleccionado por su aporte a la incorporación de los medios digitales. Para ello, se aplicó un diseño cualitativo retrospectivo con enfoque interpretativo. Los participantes fueron 34 tutores universitarios, 96 mentores de centros escolares y 315 profesores en formación de diferentes carreras de Pedagogía. La investigación se abordó desde un diseño cualitativo retrospectivo, de esta manera, es interesante la apreciación de las autoras con respecto al uso de las plataformas virtuales para la retroalimentación de los profesores de las diferentes modalidades. Aquí analizaremos las ventajas y desventajas que los docentes han encontrado en medio de nuestro contexto al cambio tecnológico del año 2020. Por otro lado, el Asimismo, Nájar y Grosso (2014) en su artículo La plataforma virtual como herramienta didáctica dinamiza la lectura y escritura, las autoras examinan como el uso de las TIC ha incrementado notoriamente la lectura y escritura de los estudiantes universitarios ya que facilita y motiva a los estudiantes y docentes a participar en discusiones que propician la construcción social del conocimiento, es decir, manifestar una opinión crítica mejorando los procesos comunicativos entre estudiantes y docentes. Podemos entonces decir que las plataformas virtuales, permiten no sólo aprender y enseñar sino también dar nuestro punto de vista en forma más dinámica dejando a un lado el interés o la valoración que el docente le otorgue. En este estudio se demuestra que con las plataformas virtuales se aumentó el interés por leer, comprender y escribir por parte de los estudiantes. Asimismo, en el artículo, Resultados de implementación de la plataforma educativa virtual Universidad Central del Ecuador elaborado por Andrade, Cadena y Ortiz (2017), podemos observar la importancia, casi dependencia, de las tecnologías de la información y comunicación. Ellos señalan que la Educación superior debe contar con espacios de interacción digital, ya que estas plataformas son espacios 
entre docentes y estudiantes, donde se facilita la comunicación, la didáctica y la pedagogía. Por último, Contreras y Garcés en su artículo, Ambientes Virtuales de Aprendizaje: dificultades de uso en los estudiantes de cuarto grado de primaria (2019), nos muestran otro lado del uso de los ambientes virtuales para el aprendizaje (AVA), en su artículo nos muestran que los recursos tecnológicos, específicamente los AVA, no han sido utilizados quizá por cierto desconocimiento, por un lado, por parte de los docentes, acerca de los requerimientos establecidos para su incorporación como parte de esa relación pedagógica: docente-alumno-entornos virtuales de aprendizaje. Y, por otro lado, por parte de los estudiantes, quienes tienen las dificultades propias de acceso a las tecnologías, aun cuando, a esta generación se le conoce como migrantes digitales. La razón de mayor peso, en los estudiantes de grado cuarto de primaria es que, en su mayoría, residen en zonas rurales, y, por tanto, no cuentan con el servicio de internet para poder apoyar su aprendizaje. Sus padres de escasos recursos económicos, no pueden tampoco proveerles de equipos tecnológicos, con los cuales puedan relacionarse con los AVA. Solamente, tienen contacto con estos recursos tecnológicos en la institución educativa y, por muy poco tiempo, por la restricción del horario.

\section{Desarrollo y discusión}

\section{Los docentes frente a las herramientas digitales.}

Las TIC configuran nuevos entornos y escenarios para la formación inicial docente en la orientación y tutorización, eliminando barreras espacio-temporales y facilitando el trabajo colaborativo y la flexibilidad en el aprendizaje (Domingo y Marqués, 2011). Algunas investigaciones buscan comprender las potencialidades del uso de las TIC en la entrega de retroalimentación formativa (Remesal, Colomina, Mauri, y Rochera, 2017) para ayudar a los estudiantes a realizar trabajos de forma colaborativa (Mauri, Ginesta \& Rochera, 2016) y en la evaluación de aprendizajes de estudiantes, favoreciendo la autorregulación en el proceso educativo docentes frente a las herramientas digitales. En tal sentido, observamos la importancia de que los docentes puedan tener las competencias necesarias no sólo para poder manejar las herramientas digitales que le permitan mantener una comunicación fluida con sus estudiantes, sino plasmar la metodología de enseñanza centrada en el alumno y con un énfasis práctico que generen aprendizajes significativos a los nuevos entornos virtuales. 
Leiva y López (2019) nos dicen que las plataformas virtuales son recursos que aportan a la organización de actividades remotas, complementando el desarrollo de una asignatura para ofrecer información y seguimiento al grupo de alumnos, lo que crea un entorno de intimidad entre profesor-aprendiz que no ofrecen otras instancias (García y Javier, 2007). El uso de las plataformas, según Boneu (2007) tiene cinco características imprescindibles: Favorecer la interactividad, flexibilidad, escalabilidad, designar roles en función del usuario, apoyar el proceso de enseñanza y aprendizaje desde la perspectiva de la planificación, estrategias metodológicas y evaluación.

De acuerdo con la sistematización de las respuestas de los sistemas educativos de América Latina a la crisis originada por la pandemia de COVID-19 realizada por el Sistema de Información de Tendencias Educativas en América Latina (SITEAL) del Instituto Internacional de Planeamiento de la Educación de la UNESCO (IIPE), en la mayoría de los países considerados (15 de un total de 18) se han proporcionado recursos de diversa índole a las y los docentes. Estos se han concentrado en la provisión de cursos en línea (11 países) y recursos en la web para docentes (9 países). Otros recursos dirigidos a docentes como parte de las respuestas educativas ante la crisis son: la provisión de bibliotecas digitales (4 países), la contención emocional (3 países) y la entrega de dispositivos digitales (2 países). (Figura 1).

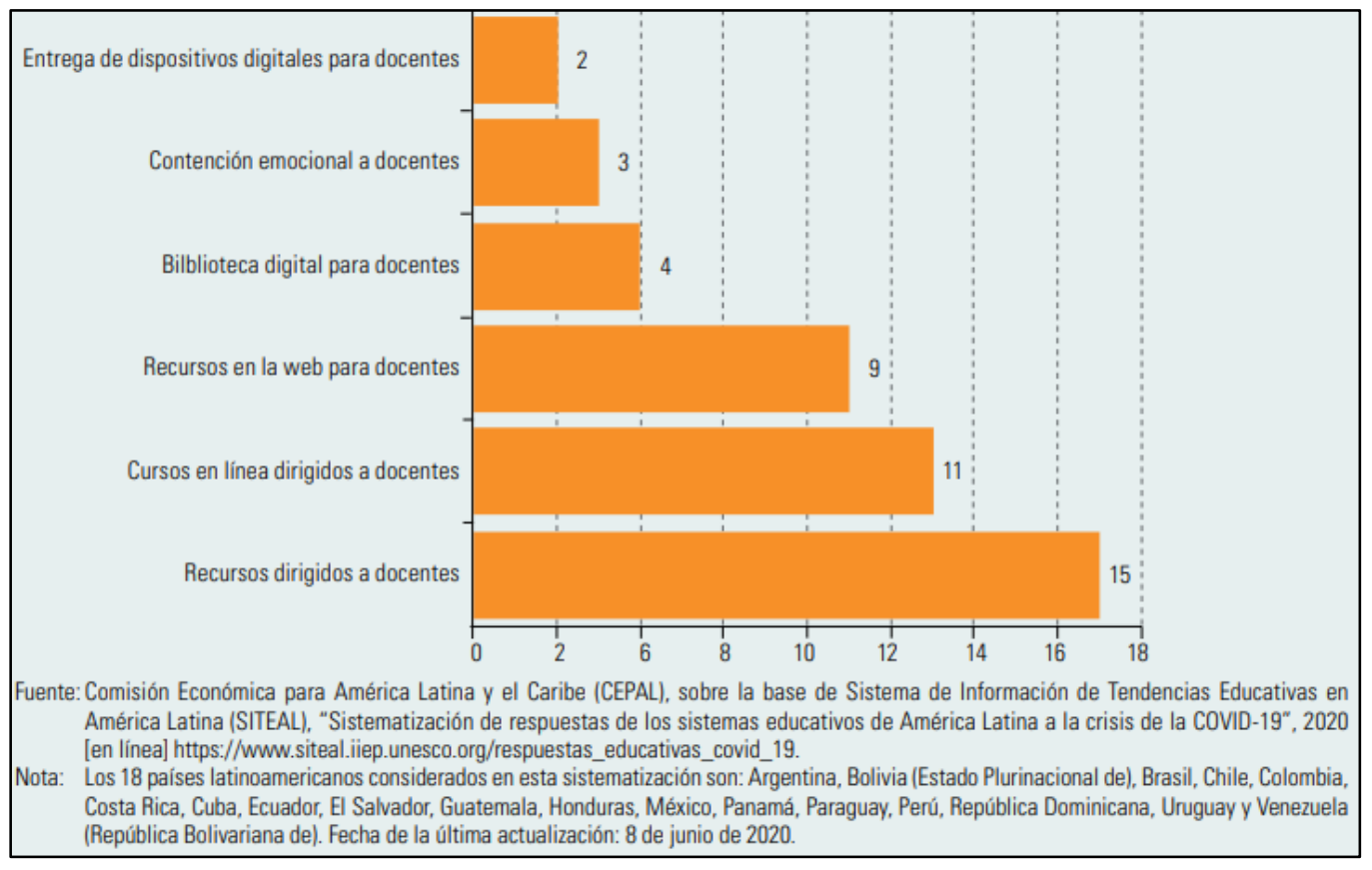

Figura 1.

América Latina (18 países): iniciativas gubernamentales de apoyo a los docentes en el marco de la crisis generada por la pandemia de COVID-19, según tipo de apoyo. 
Entre las dificultades encontradas nos dice Claro (2017), en su artículo de investigación planteó dos hipótesis para que los docentes no deseen trabajar con una plataforma informática, la primera tiene que ver a que los docentes de los cursos de informática tenían temor de utilizar esta herramienta virtual en su labor porque tendrían que trabajar en su aula de clase y aparte en su ambiente virtual y eso requería de más tiempo y trabajo y por otro lado surgía el temor de que en un futuro puedan ser reemplazados en su labor como docentes y que posteriormente pierdan su empleo.

A raíz del aislamiento 2019 por la situación pandémica vivida a nivel mundial, las instituciones educativas empezaron a llevar a través de la educación a distancia las sesiones de aprendizaje.

El Perú ha sido un país que ha pasado por la suspensión de las clases escolares por diferentes situaciones, huelga de maestros, sismos, situaciones de riesgo por infraestructura u otro motivo, pero no había experimentado el teletrabajo como medio para continuar con la educación en todo el país y esto obviamente trajo sus complicaciones al verse obligados a esta forma de trabajo a raíz de la circunstancia pandémica mundial. Entre las demandas educativas, la falta de conexión de los estudiantes, se suma la falta del conocimiento de las herramientas digitales por parte de los docentes.

Leiva y López (2019), tienen un claro concepto de la importancia del uso de las plataformas virtuales en el proceso educativo, sin embargo, hay que tomar en cuenta que es sumamente necesario en nuestro contexto, estar preparados para enfrentar los cambios que nos exigen las nuevas modalidades de aprendizaje, así como poner en práctica los conocimientos de las TIC y las TAC. Pinedo (2017) nos dice:

el acceso a una educación de calidad, es dotar al docente de herramientas que permitan complementar al contenido curricular con las tecnologías y herramientas disponibles, las cuales son un apoyo para poder presentar una clase diferente, creativa, dinámica, participativa, constructivista, e inclusivo para las estudiantes con discapacidad, de esta forma se despierta el interés de los participantes por aprender; así pues, es importante capacitar a los docentes en (TAC) para que cada educador pueda utilizar diferentes programas en sus clases, para hacerlo de forma interactiva; y podrá configurar el aula virtual de la especialidad que imparte con el objetivo de estar acorde con la tecnología. 
Vemos entonces la importancia de que el sistema educativo peruano pueda generar oportunidades y experiencias formativas en relación a las TIC, TAC, plataformas virtuales, en donde los protagonistas son una red de implicados: el Estado, mediante la formulación de políticas públicas a mediano y largo plazo, así como ver en forma amplia las diferentes necesidades de los docentes de país, tomando en cuenta nuestras diferentes realidades y realizando alianzas con las empresas que tienen que ver con las tecnologías, las universidades e institutos de educación técnica que permitan capacitar y favorecer el desarrollo profesional del docente, así como orientar en las nuevas metodologías de enseñanza, evaluación y seguimiento y por último y no menos importantes, más bien se dirían los actores principales, los directores, docentes, estudiantes, padres de familia y la comunidad educativa como los que serán quienes brindarán los servicios educativos.

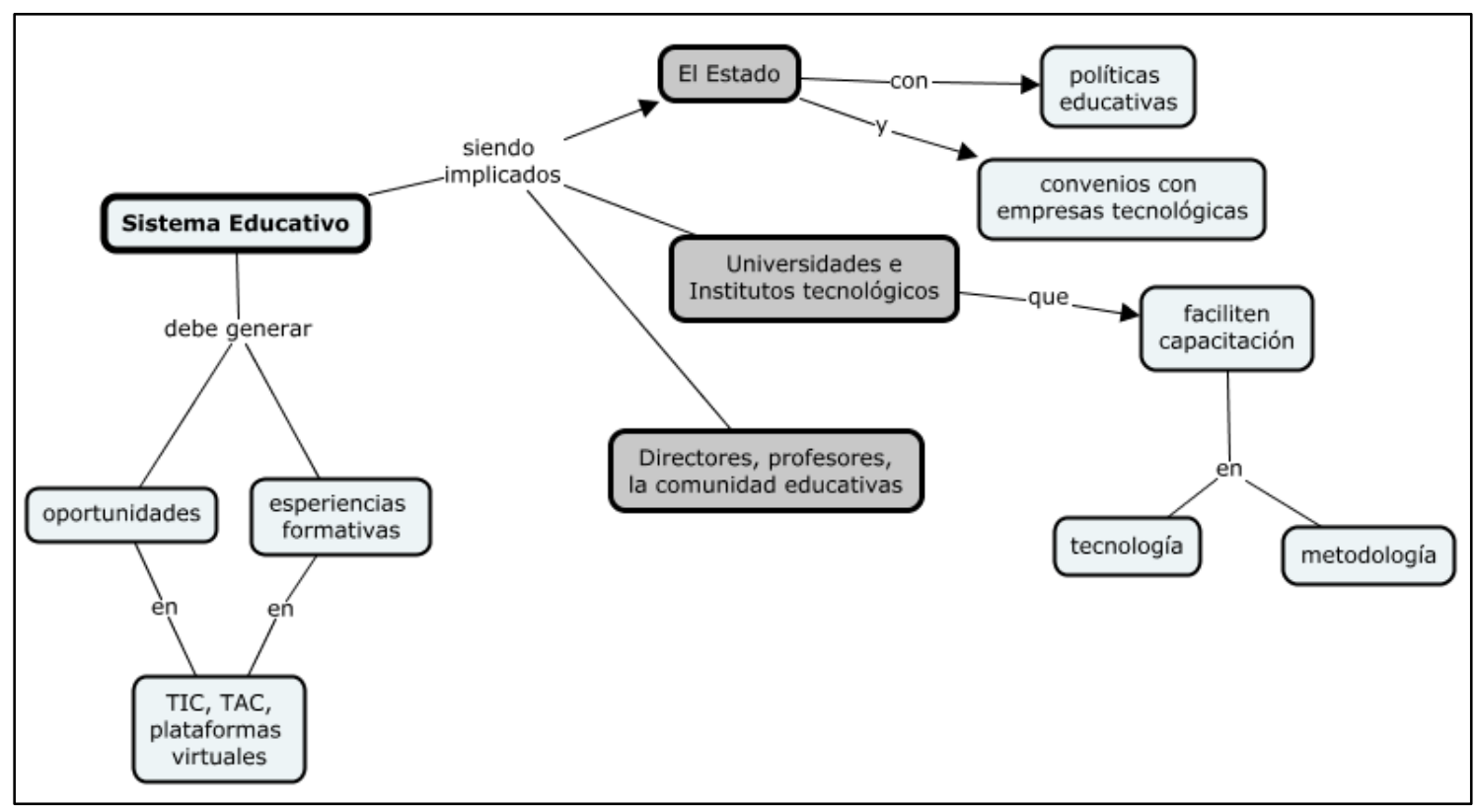

Figura 2

Los docentes frente a las herramientas digitales

\section{Las plataformas virtuales en la educación}

Claro (2017), en su artículo Valoración del uso de la plataforma virtual Moodle como recurso pedagógico en la enseñanza universitaria de la informática, nos dice que el uso de los Espacios Virtuales de Aprendizaje (EVA) son cada vez más necesarios en las instituciones educativas, en cualquier nivel y en cualquier modalidad, ya sea como un complemento para una educación semi presencial o como herramienta única en donde se desarrollen los contenidos de aprendizaje. El uso de estas herramientas de internet y de otras redes de telecomunicaciones como recursos para enseñanza son 
conocidos como e-learning. Este concepto, según Claro, incluye todos los procesos educativos tanto como planificación, enseñanza, evaluación y retroalimentación, por eso es que su manejo es provechoso y supone un conocimiento y manejo de las herramientas digitales. Claro, nos menciona también que en estos momentos hay muchas plataformas virtuales en el mercado. Tenemos las versiones libres y las de pago. En nuestro contexto la mayoría de las instituciones escolares públicas y privadas han optado por las versiones libres, tal es el caso de Edmodo, Chamilo, Moodle, Classroom, etc., todas permiten organizar estudiantes, asignar tareas, calificaciones y mantener una comunicación que involucre a profesores, estudiantes y padres de familia, además contienen aplicaciones que refuerzan las posibilidades de ejercitar destrezas intelectuales.

Según datos analizados obtenidos a partir de la Encuesta Nacional de Hogares (ENAHO 2019), a nivel regional en el Perú existen diferencias considerables en el nivel de acceso al servicio de internet. Solo tres regiones gozan de este servicio en más de la mitad de sus hogares. Se trata de Lima (56,6\%), Tacna $(52,3 \%)$ y Callao $(51,3 \%)$. En tanto, en 15 regiones el acceso a internet en los hogares oscila entre $20 \%$ y $50 \%$, destacando aquellos ubicados en la costa y selva. Los hogares menos favorecidos con el acceso al servicio (menos de 20\%) están ubicados en su mayoría en la sierra; entre ellos, los de situación más crítica son Cajamarca y Huancavelica, ya que, de cada 100 hogares, los que tiene conexión a internet son tan solo 9 y 5 hogares, respectivamente. (Figura 3).

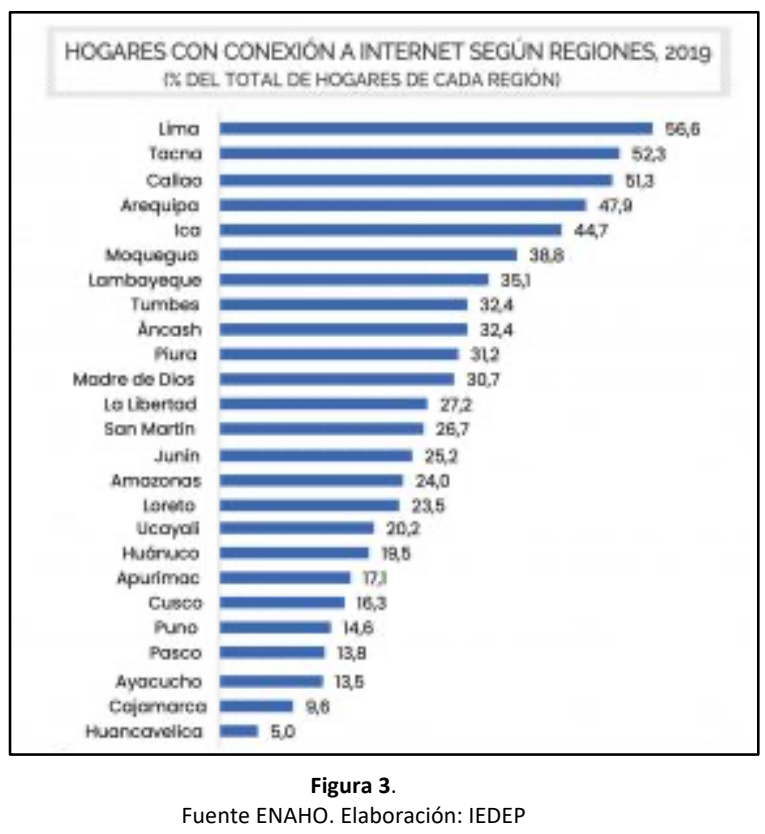


Es entonces que nos encontramos con la primera dificultad para poder emplear las plataformas virtuales, la falta de conexión de los estudiantes, el poco acceso a las herramientas digitales y la falta de conocimiento de éstas, dificultades que no son imposibles de superar y que demandarán un gran esfuerzo de toda la comunidad y este esfuerzo iniciará con los mismos docentes quienes, en muchos casos, no estaban habituados al uso de la tecnología. El logro en las mejoras de los resultados dependerá en gran medida de la habilidad del docente para estructurar el ambiente de aprendizaje, según la Unesco, 2008, (Hernandez 2017), tendremos que dar el "salto" y "romper" esquemas tradicionales con un aprendizaje basado en la cooperación y el trabajo en equipo, tomando en cuenta las plataformas virtuales que tendremos a nuestro alcance.

Andrade y Cadena (2017) en su artículo Resultados de implementación de la plataforma educativa virtual en la Universidad Central del Ecuador cuyo objetivo fue determinar la Plataforma Educativa Virtual a utilizar, los resultados obtenidos en la implementación y la descripción del desarrollo de la actividad del docente y el estudiante. Entre las conclusiones a las que llegaron, destacaron que la implementación de la plataforma no tuvo inconvenientes técnicos, pues se contó con una infraestructura de tecnológica sólida en la universidad donde aplicaron la investigación, así mismo determinaron la plataforma Moodle como la que presta mejor servicio, por su usuabilidad, soporte y adaptibilidad. Por otro lado, las herramientas más usadas por los estudiantes son los foros, cuestionarios, los archivos, mientras que para los docentes es la creación de cursos, su organización y tareas. Y finaliza recomendando la necesidad de diseñar un Plan de Capacitación Continua para el docente con el propósito de establecer acciones de mejora continua.

Pérez y Saker (2013) en su artículo Efectividad del uso de la plataforma virtual en el proceso de enseñanza y aprendizaje en la Universidad del Magdalena, investigaron sobre las ventajas, desventajas y limitaciones de la implementación de didácticas apoyadas en el uso de plataformas tecnológicas para asegurar aprendizajes duraderos. Ellos observaron que los estudiantes tienen la necesidad de adquirir computadoras portátiles o de escritorio o teléfonos móviles y utilizan el servicio de Internet fijo o móvil, como herramientas necesarias para desarrollar sus estudios universitarios, aunque, hay un pequeño grupo de estudiantes que no cuentan con ninguna de estas herramientas por no tener los recursos económicos necesarios, pero pueden alquilar máquinas que les ofrezcan ayuda para culminar sus estudios, por otro lado el $97 \%$ de los estudiantes recibió enseñanza de Informática antes de ingresar a la Universidad, lo que 
les da ciertas ventajas a los estudiantes del programa de Informática. Asimismo, el grueso grupo de estudiantes les gustaría complementar el desarrollo de asignaturas disciplinares mediante el uso de una plataforma virtual y del Internet, que les brinde otras posibilidades en cuanto a las lecturas, documentos, descargar libros y materiales, pero sobre todo intercambiar ideas, opiniones, trabajos de manera colaborativa, etc. Como vemos, fue muy ventajoso el uso de las herramientas virtuales desde mucho antes que pasara el aislamiento por el COVID19, muchas instituciones se preparaban a un entorno remoto por varios motivos, uno de ellos fue el tiempo y el bajo costo.

Nájar y Grosso (2014), nos mencionan en su artículo La plataforma virtual como herramienta didáctica dinamiza la lectura y escritura. Los autores concluyen que la plataforma virtual definitivamente da facilidades y motiva a estudiantes y docentes a participar en diálogos que propician la construcción de conocimiento y esto refleja que el estudiante ha leído, dando lugar al incremento significativo al interés por leer, comprender y escribir. Se evidencio que los estudiantes aseguran que tanto el trabajar en la plataforma como las estrategias y talleres empleados por el docente contribuyen a disminuir la brecha digital con la que ellos llegan a la universidad.

Por otro lado, Contreras y Garcés (2019), nos refiere en su artículo Ambientes Virtuales de Aprendizaje: dificultades de uso en los estudiantes de cuarto grado de primaria, que, para el desarrollo de las actividades de aprendizaje en un entorno virtual, es necesario que ellos entiendan y comprendan cada uno de los pasos que se llevarán a cabo en el trabajo que se va a realizar; además de tener conocimiento sobre la plataforma a utilizar. En este caso los estudiantes de cuarto grado de primaria no poseen habilidades de autoaprendizaje para desarrollar actividades en un ambiente virtual, además que no toman iniciativas en la búsqueda de estrategias que contribuyan a la solución de los inconvenientes que se les presente, sin embargo, los estudiantes que sí logran estas habilidades se convierten en mediadores de sus pares al momento de hacer sus preguntas o al opinar sobre lo que el docente presente. Aún con todo, los autores también manifiestan que los estudiantes que han tenido la oportunidad de interactuar con la virtualidad son más efectivos en el uso de dichos espacios y por ende su desempeño personal en el logro de competencias es más significativo, tomando en cuenta que los AVA, como aplicación informática, no suponen en sí mismas un desarrollo del potencial pedagógico, sino que se toma como un recurso tecnológico que viene a apoyar y a enriquecer los procesos de enseñanza aprendizaje para el logro de su formación integral. 
En la modalidad asíncrona de enseñanza el estudiante tiene que adquirir nuevas competencias, no sólo las tecnológicas y superar las barreras de las señales de internet sino el que adquiera un hábito de estudios diferente. El artículo de la UNESCO (2017) Docentes y sus aprendizajes en su modalidad virtual, menciona que el estudiante tiene que al estudiar en las plataformas virtuales debe autorregular el ritmo de estudio, el tiempo y su espacio, esto genera un alto grado de autonomía, el estudiante es el que toma todo el control de su aprendizaje por medio de los materiales que el docente ya ha preparado. Esto genera independencia, el estudiante elegirá qué ritmo y estilo de aprendizaje tomará dentro de los parámetros que ofrecerá la organización educativa.

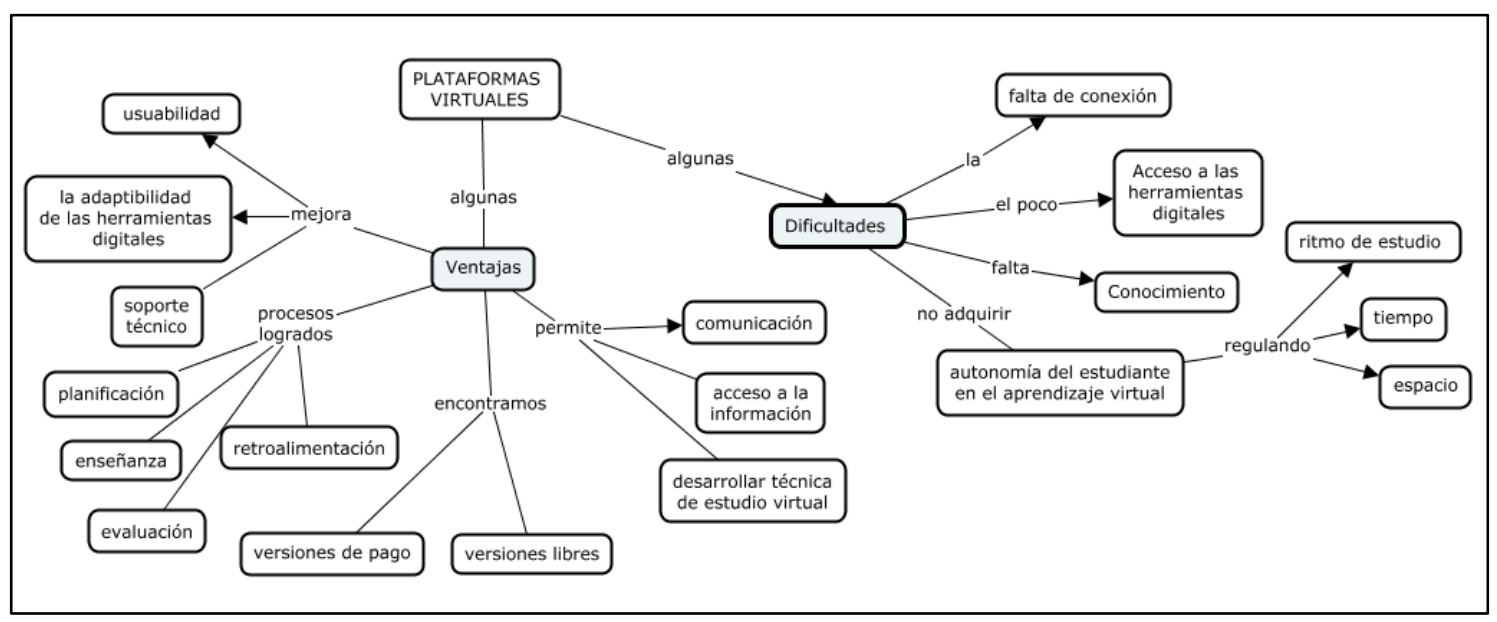

Figura 4.

Las plataformas virtuales en la educación

Las plataformas virtuales, entonces, contribuyen al logro de competencias siempre y cuando los estudiantes cuenten con conocimiento e información. Por otro lado, mientras en algún momento fue un recurso opcional, hoy en día en medio de esta pandemia y los diferentes cambios que hay en cuanto a los confinamientos por cuarentena, se ha hecho indispensable tener el conocimiento técnico, variar la metodología e ir capacitándonos en los diferentes recursos que se nos presentan de acuerdo a la especialidad y modalidad educativa.

\section{Conclusiones}

En los tiempos que estamos viviendo debemos replantear el esquema metódico y aislado a la tecnología en la que hemos permanecido y que seguramente tiene sus ventajas y desventajas, pero tenemos que considerar que la metodología de enseñanza 
debe adaptarse a las circunstancias, al ritmo de aprendizaje, al tiempo y al espacio. Es por ello, que el docente, sea de la modalidad en la que se encuentre, debe adquirir conocimientos de las TIC y las TAC para poder emplear las plataformas virtuales que son, al menor por ahora, el único medio de contacto con los estudiantes.

Las plataformas virtuales son un medio indispensable para la enseñanza aprendizaje, los diferentes medios de comunicación se basan en las nuevas formas de trabajo remoto y asíncrono, los centros laborales, las instituciones educativas, etc., basan sus logros y avances en esta forma de trabajo que se ha vuelto indispensable para toda sociedad.

Finalmente, hemos comprobado a través de la presentación de diferentes artículos, que las plataformas virtuales son una herramienta necesaria y de mucha utilidad para la enseñanza aprendizaje remota, ya que facilita el planificación, enseñanza, evaluación y retroalimentación, procesos necesarios tanto para el docente como para el estudiante (Tomalá, Gallo, Mosquera y Chancusig, 2020), esto conlleva a tener conocimientos y aprendizajes de las nuevas tecnologías y actualizarse constantemente.

\section{Referencias Bibliográficas}

Andrade M. , Cadena S., Ortiz J.(2017) Resultados de implementación de la plataforma educativa virtual Universidad Central del Ecuador. Recuperado de: file://C:/Users/Lenovo/Downloads/76-Texto\%20de1\%20art\%C3\%ADculo-228-210-20190130\%20(1).pdf

Cervantes, Mónica Luz (2013) Efectividad del uso de la plataforma virtual en el proceso de enseñanza y aprendizaje en la Universidad del Magdalena. Revista de

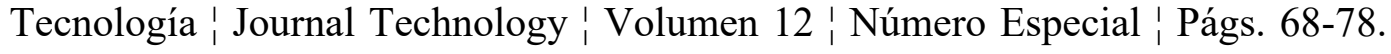
Colombia.

Cabero J., Marín V. y Castaño C. (2015). Validación de la aplicación del modelo TPACK para la formación del profesorado en TIC. Revista de innovación educativa. Barcelona. España. Recuperado de: https://www.researchgate.net/publication/279207022_Validacion_de_la_aplicacio n del modelo TPACK para la formacion del profesorado en TIC

Claro, Juan Manuel (2017). Valoración del uso de la plataforma virtual Moodle como recurso pedagógico en la enseñanza universitaria de la informática. Perspectivas 
Volumen 2 (1) Enero - Junio de 2017, páginas 43 - 56. Universidad de Santander (UDES), Cúcuta. Colombia.

Coll C., Monereo C. (2008) Educación y aprendizaje en el siglo XXI: Nuevas herramientas, nuevos escenarios, nuevas Finalidades, Psicología de la educación virtual. Ediciones Morata, S. L. Fundada por Javier Morata, Editor, en 1920 C/ Mejía Lequerica, 12 - 28004 - MADRID.

Contreras A. y Garcés L. (2019). Ambientes Virtuales de Aprendizaje: dificultades de uso en los estudiantes de cuarto grado de primaria. Prospectiva. Revista de Trabajo Social e intervención social No. 27, enero-junio 2019: pp. 215-240. Universidad del Valle. Universidad Pública, Cali-Colombia. Recuperado de: http://www.scielo.org.co/pdf/prsp/n27/2389-993X-prsp-27-00215.pdf

Cooperberg (2002). Las herramientas que facilitan la comunicación y el proceso de enseñanza-aprendizaje en los entornos de educación a distancia. Revista de Educación a distancia RED. Recuperado de: https://revistas.um.es/red/article/view/25211

Domingo M. Marquéz P. (2011) Aulas 2.0 y uso de las TIC en la práctica docente. Comunicar, $n^{\circ}$ 37, v. XIX, 2011, Revista Científica de Educomunicación; ISSN: 1134-3478; páginas 169-175. a Facultad de Educación de la Universitat Internacional de Catalunya y de la Facultad de Pedagogía de la Universidad de Barcelona. España. Recuperado de: file://C:/Users/Lenovo/Downloads/10.3916_C37-2011-03-09.pdf

ENAHO (2019), Perú - Encuesta Nacional de Hogares sobre Condiciones de Vida y Pobreza.

García A. y Javier F. (2007). Estudio de las actitudes del profesorado universitario hacia la integración de las TIC en su práctica docente. $10^{\circ}$ Congreso Iberoamericano EDUTEC 2007, 23-25 Octubre, Buenos Aires (Argentina). Universidad de Salamanca, España. Recuperado de: https://gredos.usal.es/bitstream/handle/10366/18450/DDOMI_Estudiodelas.pdf?se quence $=1$

Garcia F. (2017). Competencias digitales en la docencia universitaria del siglo XXI., Facultad de Educación, Departamento de Didáctica y Organización Escolar 
Miryam Maria del Rosario Ramos Vite, Luz Carola Macahuachi Nuñez de Castillo

Universidad Complutense de Madrid-España. Recuperado: https://eprints.ucm.es/id/eprint/44237/

Guerrero, Maria (2019). Uso de plataforma virtual para retroalimentar la formación del profesorado. Pensamiento Educativo. Revista de Investigación Educacional Latinoamericana 2019, 56(2), 1-17. Pontificia Universidad Católica de Chile. Chile. Recuperado de: https://pensamientoeducativo.uc.cl/files/journals/2/articles/1144/public/11445265-3-PB.pdf

Hernandez R., (2017). Impacto de las TIC en la educación: Retos y Perspectivas. Revista USIL, Propósitos y representaciones, revista de psicología educativa. Universidad San Ignacio de Loyola, Lima, Perú. Recuperado de: https://revistas.usil.edu.pe/index.php/pyr/article/view/149/381

Leiva y López (2019), Uso de plataforma virtual para retroalimentar la formación del profesorado Pensamiento Educativo. Revista de Investigación Educacional Latinoamericana 2019, 56(2), 1-17. Pontificia Universidad Católica de Chile. Recuperado de: https://pensamientoeducativo.uc.cl/files/journals/2/articles/1144/public/11445265-3-PB.pdf

Mauri A., Ginesta R., y Rochera T., (2016). Uso de cuestionarios online con feedback automático para la e-innovación en el alumnado universitario. Red de Revistas Científicas de América Latina, el Caribe, España y Portugal. Recuperado: https://www.redalyc.org/pdf/158/15850360005.pdf

May N., Patrón R., y Sahuí J. (2017). La capacitación como estrategia para combatir las barreras tecnológicas y de actitud de los trabajadores de las Mipymes en México. Revista electrónica del desarrollo humano para la innovación social. Mexico. Recuperado de: https://www.cdhis.org.mx/index.php/CAGI/article/view/115

Monzoy E., Acosta D. y Sanchez C. (2011). La capacitación docente, factor clave en la calidad de la educación superior en el Instituto Tecnológico de Oaxaca. RIDE Revista Iberoamericana para la Investigación y el Desarrollo Educativo E-ISSN: 2007-7467. Recuperado de: https://www.redalyc.org/pdf/4981/498150310006.pdf

Muñoz L., García L. y Rodriguez M. (2011). Revista Lasallista de Investigación. Revista Lasallista de Investigación, vol. 9, núm. 1, 2012, pp. 53-61 Corporación 
Miryam Maria del Rosario Ramos Vite, Luz Carola Macahuachi Nuñez de Castillo

Universitaria Lasallista Antioquia, Colombia. Recuperado de: https://www.redalyc.org/pdf/695/69524955003.pdf

Najar O. y García S. (2014). La plataforma virtual como herramienta didáctica dinamiza la lectura y la escritura. Revista Vínculos, (2014), 11 (1). Universidad Distrital Francisco José de Caldas. Colombia. Recuperado de:file://C:/Users/Lenovo/Downloads/8025-texto\%20del\%20art\%C3\%ADculo37545-1-10-20141219.pdf

OCDE (2015), Organización para la Cooperación y el Desarrollo Económicos, París. Revista Perspectivas de la OCDE sobre la economía digital 2015. Recuperado de: http://www.oecd.org/sti/ieconomy/DigitalEconomyOutlook2015_SP_WEB.pdf

Perez M., Saker A. (2013), Efectividad del uso de la plataforma virtual en el proceso de enseñanza y aprendizaje en la Universidad del Magdalena. Revista de Tecnología | Journal Technology | Volumen 12 | Número Especial | Págs. 68-78. Colombia.

Puentes A., Roig R., Sanhueza S., Friz M. (2013). Concepciones sobre las Tecnologías de la Información y la Comunicación (TIC y sus implicaciones educativas: Un estudio exploratorio con profesorado de la provincia de Nuble, Chile . Recuperado de: https://www.redalyc.org/pdf/924/92425714004.pdf

Remesal A., Colomina R., Mauri T., y Rochera M., (2017). Uso de cuestionarios online con feedback automático para la e-innovación en el alumnado universitario. Comunicar- Revista científica de comunicación y Educación. Desarrollo y Psicología de la Educación de la Universidad de Barcelona. Recuperado: file://C:/Users/Lenovo/Downloads/10.3916-C51-2017-05.pdf

REDIECH, vol. 10, núm. 19, 2019, Chihuahua, México. Recuperado de: https://www.redalyc.org/jatsRepo/5216/521658239005/html/index.html

Resolución Viceministerial Nº97-2020-MINEDU

SITEAL, Sistema de información de tendencias educativas en América Latina. Recuperado de: https://www.siteal.iiep.unesco.org/

Tarango J. (2019), Evaluación según diseño y aprendizaje de Google Classroom y Chamilo, Revista de Investigación Educativa de la 
Tomalá M., Gallo G., Mosquera J. y Chancusig J. (2020). Revista científica mundo de la investigación y el conocimiento Vol 4 Nro. 4. Guayaquil. Ecuador. Recuperado de: https://recimundo.com/index.php/es/article/view/899/1436 